\title{
LINEAR STABILITY OF A PLANE FRONT IN SOLID COMBUSTION AT LARGE HEAT OF REACTION*
}

\author{
FERNANDO VARAS AND JOSÉ M. VEGA
}

\begin{abstract}
The existence and linear stability of a uniformly propagating plane front in solid combustion are considered under the assumption of a large heat of reaction, which is of interest in pyrotechnic mixtures. The Zel'dovich number is not necessarily large, and the chemical reaction is not confined to a reaction sheet for typical values of the parameters. Thus the effective calculation of the fronts and the analysis of their stability rely on qualitative methods and on some numerics. It is seen that there is always a minimum burning rate and that the front is stable only if the heat of reaction is sufficiently large. A comparison is made with analytical results obtained from a phenomenological delta-function model.
\end{abstract}

Key words. solid combustion, pyrotechnic mixtures, delay detonators, plane fronts

AMS subject classifications. 35K57, 80A25, 80A32

PII. S0036139901386417

1. Introduction. This paper deals with the linear stability of plane fronts in solid combustion at large activation energy and large reaction heat, such that the Zel'dovich number is not necessarily large. This limit is of interest in wave propagation in pyrotechnic mixtures (see [1] and references given therein), which are frequently used in delay detonators [2].

If reactant diffusion and heat losses are ignored, then the conservation equations in dimensional form are

$$
\begin{aligned}
& \rho c_{p} \frac{\partial \tilde{T}}{\partial \tilde{t}}-\kappa \tilde{\Delta} \tilde{T}=Q A \tilde{c}^{n} \exp \left(\frac{-E}{R \tilde{T}}\right), \\
& \frac{\partial \tilde{c}}{\partial \tilde{t}}=-A \tilde{c}^{n} \exp \left(\frac{-E}{R \tilde{T}}\right)
\end{aligned}
$$

in $-\infty<\tilde{x}<\infty,-\infty<\tilde{y}<\infty$, with boundary conditions

$$
\begin{aligned}
& \tilde{T}=\tilde{T}_{i}, \quad \tilde{c}=\tilde{c}_{i} \quad \text { as } \tilde{x} \rightarrow-\infty, \quad \tilde{T}=\tilde{T}_{f}, \quad \tilde{c}=0 \quad \text { as } \tilde{x} \rightarrow \infty, \\
& \tilde{T} \text { and } \tilde{c} \text { bounded as }|\tilde{y}| \rightarrow \infty,
\end{aligned}
$$

where the usual notation is employed. $\tilde{\Delta}$ is the Laplacian operator in terms of dimensional space variables; $\tilde{T}_{i}$ and $\tilde{c}_{i}$ are the "initial" temperature and reactant concentration, assumed to be such that the chemical reaction is frozen at the cold boundary but not at the flame, which requires that

$$
\frac{\beta \tilde{T}_{f}}{\tilde{T}_{i}} \equiv \frac{E\left(\tilde{T}_{f}-\tilde{T}_{i}\right)}{R \tilde{T}_{i} \tilde{T}_{f}} \gg 1,
$$

${ }^{*}$ Received by the editors March 15, 2001; accepted for publication (in revised form) January 14, 2002; published electronically June 12, 2002. This research was supported by DGES under grants PB96-0341 and PB97-0556.

http://www.siam.org/journals/siap/62-5/38641.html

$\dagger$ E.T.S.I. de Minas, Universidad de Vigo, Lagoas-Marcosende, 36280-Vigo, Pontevedra, Spain (fvaras@uvigo.es).

‡E.T.S.I. Aeronáuticos, Universidad Politécnica de Madrid, Plaza Cardenal Cisneros 3, 28040Madrid, Spain (vega@fmetsia.upm.es). 
where $\beta=E\left(\tilde{T}_{f}-\tilde{T}_{i}\right) /\left(R \tilde{T}_{f}^{2}\right)$ is the Zel'dovich number; and $\tilde{T}_{f}$ is the "final" temperature, which is assumed to be such that the total (chemical + thermal) enthalpy is conserved across the front, namely,

$$
Q \tilde{c}_{i}+\rho c_{p} \tilde{T}_{i}=\rho c_{p} \tilde{T}_{f} .
$$

Thus condition (1.5) is satisfied either if the Zel'dovich number is large (the usual limit) or if

$$
\frac{\tilde{T}_{f}}{\tilde{T}_{i}} \gg 1
$$

which requires that the heat of reaction be appropriately large (namely, $Q \tilde{c}_{i} /\left(\rho c_{p} \tilde{T}_{i}\right) \gg$ 1) according to (1.6), and which is the limit considered below. In typical pyrotechnic mixtures [1] the activation energy ranges from 13 to $23 \mathrm{~kJ} \mathrm{~mol}^{-1}$, with final temperatures on the order of $1,800^{\circ} \mathrm{K}$. Then, for an ambient temperature of $300^{\circ} \mathrm{K}$, the ratios $E /\left(R \tilde{T}_{i}\right)$ and $E /\left(R \tilde{T}_{f}\right) \sim \beta$ range from 5.20 to 9.25 and from 0.86 to 1.54 , respectively; thus the assumptions (1.5) and (1.7) hold. Note in particular that the Zel'dovich number can hardly be considered as large.

Conditions (1.5) and (1.6) are necessary to allow (approximately) uniformly propagating fronts.

If the temperature, the reactant concentration, the space and time variables, and the heat of reaction are nondimensionalized as

$$
\begin{gathered}
T=\frac{R \tilde{T}}{E}, \quad c=\frac{\tilde{c}}{\tilde{c}_{i}}, \quad(x, y)=\left(\frac{Q E A c_{i}^{n}}{\kappa R}\right)^{1 / 2}(\tilde{x}, \tilde{y}), \\
t=\frac{Q E A c_{i}^{n}}{\rho c_{p} R} \tilde{t}, \quad q=\frac{R Q \tilde{c}_{i}}{E \rho c_{p}}
\end{gathered}
$$

and assumptions (1.5)-(1.6) are taken into account, then (1.1)-(1.4) are rewritten as

$$
\frac{\partial T}{\partial t}-\Delta T=c^{n} \exp \left(\frac{-1}{T}\right), \quad \frac{\partial c}{\partial t}=-q^{-1} c^{n} \exp \left(\frac{-1}{T}\right)
$$

in $-\infty<x<\infty,-\infty<y<\infty$, with boundary conditions

$$
\begin{aligned}
& c=1, \quad T=0 \text { at } x=-\infty, \quad c=0, \quad T=q \quad \text { at } x=\infty, \\
& T \text { and } c \text { bounded as }|y| \rightarrow \infty,
\end{aligned}
$$

where the boundary condition $T=0$ at the cold boundary comes from assumption (1.5); thus (1.9)-(1.11) does not exhibit the cold boundary difficulty [3]. That problem depends only on the nondimensional parameter $q$, which can be seen as a nondimensional heat release and, according to (1.6), can also be written as

$$
q=\frac{R\left(\tilde{T}_{f}-\tilde{T}_{i}\right)}{E}=\frac{\left(1-\tilde{T}_{i} / \tilde{T}_{f}\right)^{2}}{\beta},
$$

in terms of the Zel'dovich number $\beta$ defined above. The evolution problem (1.9)-(1.11) could always be treated numerically by an adaptive method to efficiently resolve the moving thin reaction region if $q$ is small [4].

In the limit $q \ll 1$, the Zel'dovich number is large, and large-activation-energy asymptotics applies. This limit was considered by Matkowsky and Sivashinsky [5] for 
first order reactions in the restricted one-dimensional (1-D) case through the following model:

$$
\begin{gathered}
\frac{\partial u}{\partial \tau}=\frac{\partial^{2} u}{\partial \xi^{2}}-\frac{\partial u}{\partial \xi} \quad \text { if } \xi \neq \phi, \\
u=0 \quad \text { at } \xi=-\infty, \quad[u]=0, \quad-\left[\frac{\partial u}{\partial \xi}\right]=1+\phi^{\prime}=\exp \left(\frac{u-1}{2 q}\right) \quad \text { at } \xi=\phi,
\end{gathered}
$$

where $[f]=f\left(\xi^{+}, \eta, \tau\right)-f\left(\xi^{-}, \eta, \tau\right)$ denotes the jump across the reaction sheet, which is located at $\xi=\phi(\tau)$. This model gives the solution in the preheated zone, which is such that the new variables

$$
u=\frac{T}{q}, \quad \xi=x \exp \left(\frac{1}{2 q}\right)-t, \quad \text { and } \quad \tau=t \exp \left(\frac{1}{q}\right)
$$

are of order unity. The reactant concentration is given by $c=1$ if $\xi<\phi$, and $c=0$ if $\xi>\phi$. The jump condition across the reaction sheet results from matching conditions with the (unique) steady state of the appropriate 1-D rescaled (according to $1-u \sim \xi-\phi \sim q$ and $c \sim 1$ ) version of (1.9)-(1.11) that applies in the reaction zone; see (3.8)-(3.9) below. This quasi-steady approximation in the reaction zone relies on the implicit assumption that the steady solution in this zone is asymptotically stable, for otherwise small perturbations in this zone will grow exponentially in its characteristic time-scale, $\tau \sim q^{-2}$, which in fact is much shorter than that considered above. This assumption fails, as can be anticipated from well-known results of Peters [6] (see also $[7,8,9])$ on the stability of premixed reaction zones for finite Lewis numbers. In fact, plane fronts have been recognized as inherently unstable in solid combustion [3] for infinite activation energy. Matkowsky and Sivashinsky considered a finite activation energy in (1.12)-(1.13), which can be considered a phenomenological model, sometimes called a delta-function model [10], for large-but-finite Zel'dovich numbers; see [11] for a discussion of this model. In fact, this model yields results that are in good qualitative agreement with some experiments [5]; a further comparison is made below. Here we will just mention that (1.12)-(1.13) has a unique steady state, which corresponds to a traveling front of (1.9)-(1.11) with a velocity (see (1.14)) $v=\exp [-1 /(2 q)]$ in a first approximation. The normal mode solutions of the linearized problem around this steady state lead to the following characteristic equation (see [5]):

$$
\tilde{\lambda}\left[16 q^{2} \tilde{\lambda}^{2}+\left(4 q^{2}+8 q-1\right) \tilde{\lambda}+2 q\right]=0,
$$

which gives the rescaled (according to (1.14)) growth rate $\tilde{\lambda}$ in terms of $q$. The root $\tilde{\lambda}=0$ results from invariance under translations, and the remaining two roots yield an oscillatory instability at the instability limit

$$
q=q_{c}=\frac{1}{2(2+\sqrt{5})} \simeq 0.118,
$$

with an eigenfrequency $\tilde{\omega}_{c}=\left(8 q_{c}\right)^{-1 / 2} \simeq 1.03$; the front is linearly stable if $q \geq q_{c}$ and unstable otherwise. All these agree qualitatively with the exact results obtained numerically below, despite the fact that this can be seen as only a phenomenological model. Additionally, as $q \rightarrow 0$, when the model really applies, (1.15) yields two unstable real eigenvalues, namely,

$$
\tilde{\lambda}=2 q+O\left(q^{2}\right) \quad \text { and } \quad \tilde{\lambda}=\frac{1}{16 q^{2}}+O\left(\frac{1}{q}\right)
$$


of which the first can make sense as an approximation of an eigenvalue of the original problem (see below) but the second is spurious because its order of magnitude is such that the associated solution in the reaction zone (implicit in the model (1.12)-(1.13)) cannot be considered as quasi-stationary; in fact, there is an eigenmode whose growth rate behaves as in the second equality in (1.16), but it is completely associated with the dynamics in the reaction zone; see below.

For further comparison, it is convenient to consider the extension of (1.12)-(1.13) to higher dimensions. Such an extension would consist of (i) allowing $u$ and $\phi$ to depend on transversal variables

$$
\eta=y \exp \left(\frac{1}{2 q}\right) \quad \text { and } \quad \zeta=z \exp \left(\frac{1}{2 q}\right),
$$

(ii) replacing $\partial^{2} u / \partial \xi^{2}$ by the Laplacian operator in (1.12), and (iii) replacing $\partial u / \partial \xi$ and $1+\phi^{\prime}$ in (1.13) by the derivative of $u$ along the unit normal to the reaction sheet (pointing towards the burnt side) and replacing $1+\phi^{\prime}$ by the normal velocity of the reaction sheet. The characteristic equation (1.15) must be replaced by the dispersion relation

$$
(4 \tilde{\lambda} q+1) \sqrt{1+4\left(\tilde{\lambda}+\tilde{k}^{2}\right)}=2 \tilde{\lambda}+1
$$

in terms of the rescaled (according to (1.17)) transversal wavenumber $\tilde{k}$, which coincides modulo notation differences with that in $[10$, p. 74$]$ and yields two branches, namely, (a) a real branch satisfying $\tilde{\lambda}<0$ if $\tilde{k}>0, \tilde{\lambda} \sim-\tilde{k}^{2} /(2 q)$ as $\tilde{k} \rightarrow 0$, which corresponds to the zero eigenvalue of (1.15), and (b) two complex conjugate branches, which yield an oscillatory instability at the marginal instability curve, with an eigenfrequency $\tilde{\omega}$ that is also indicated,

$$
q=\frac{1+4 \tilde{k}^{2}}{2\left[2+6 \tilde{k}^{2}+\left(1+2 \tilde{k}^{2}\right) \sqrt{5+16 \tilde{k}^{2}}\right]}, \quad \tilde{\omega}=\sqrt{\frac{1+4 \tilde{k}^{2}}{8 q}} .
$$

These are plotted (and labelled BL) in Figure 1.

Against this background, the main object of this paper is to analyze the plane fronts of (1.9)-(1.11) and their linear stability for finite $q$, without further assumptions or approximations. For simplicity we consider below only first order reactions, namely, we take $n=1$, but the results below are extended, somewhat straightforwardly, for $n>-1$. Note that if $n \leq 0$, the right-hand sides of the first two equalities in (1.9) must be replaced by zero when $c=0$, and if $n<1$, then the relevant solutions are such that $c=0$ if $x>\bar{x}(y, t)$, where the position of the free boundary $\bar{x}(y, t)$ must be determined as a part of the solution.

2. Uniformly propagating plane fronts. Now we seek solutions of (1.9)(1.11) of the form

$$
T=T(\xi), \quad c=c(\xi) \quad \text { with } \xi=x+v t,
$$

where the burning rate $v$ must be determined as a part of the solution. Substitution of (2.1) into (1.9)-(1.11) yields

$$
\begin{aligned}
& T^{\prime \prime}-v T^{\prime}=-c \exp \left(\frac{-1}{T}\right), \quad v c^{\prime}=-q^{-1} c \exp \left(\frac{-1}{T}\right) \quad \text { in }-\infty<\xi<\infty, \\
& T=0, \quad c=1 \quad \text { at } \xi=-\infty, \quad T=q, \quad c=0 \quad \text { at } \xi=\infty .
\end{aligned}
$$


(a)

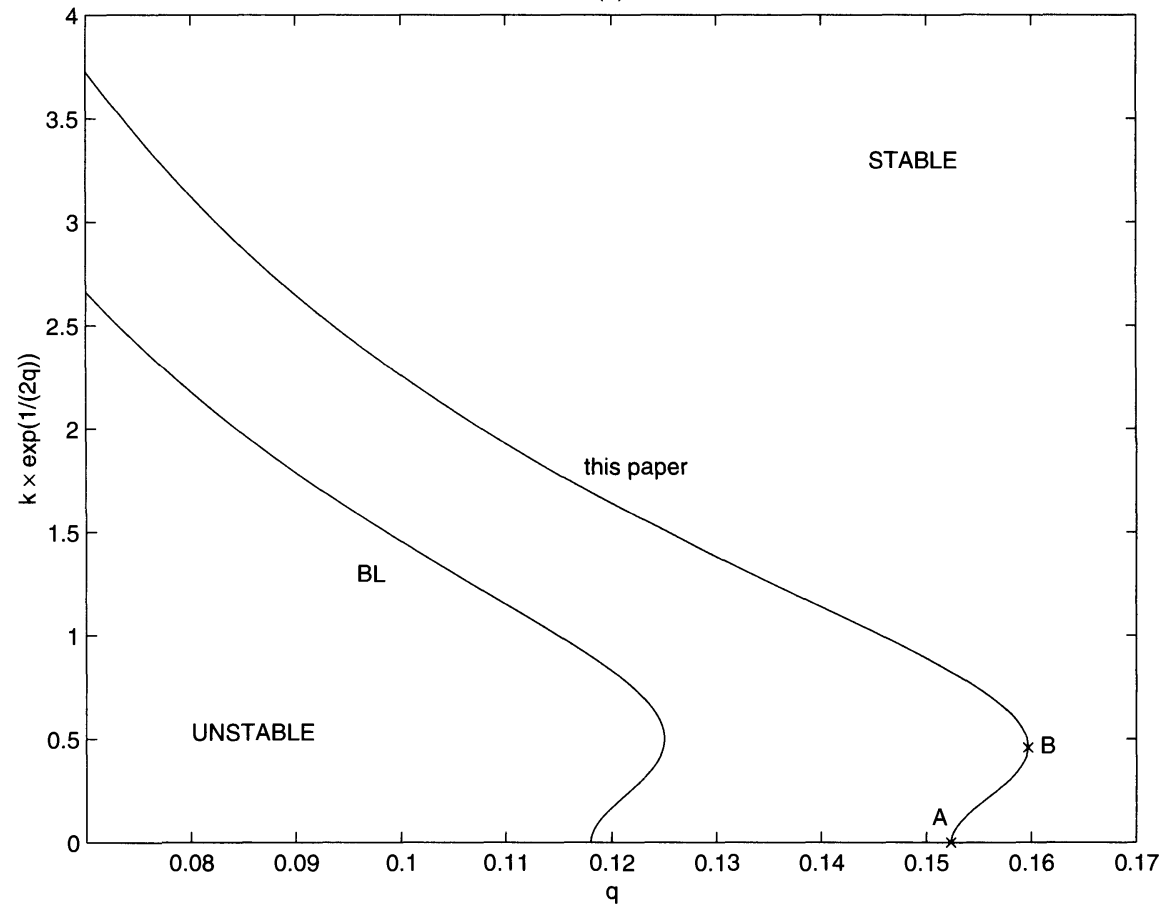

(b)

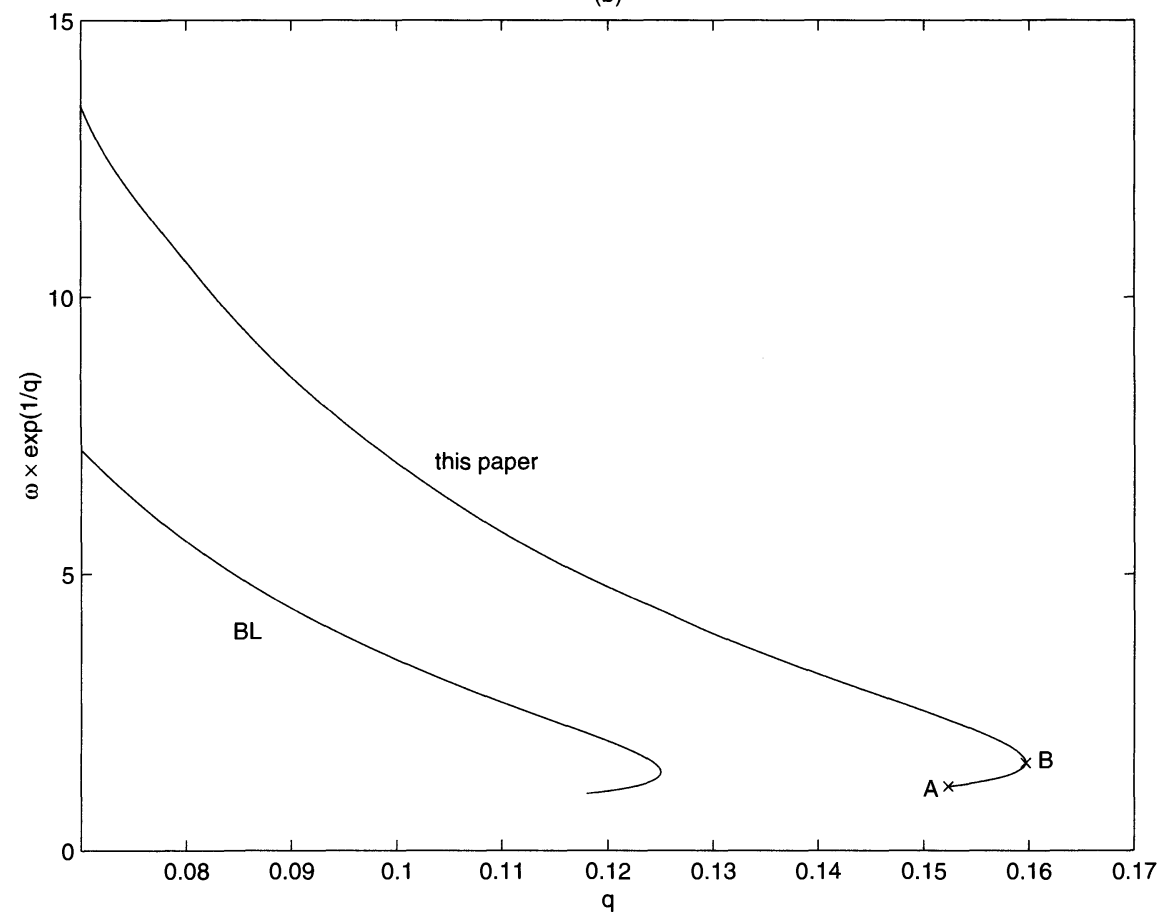

Fig. 1. Neutral instability curve (a) and the associated eigenfrequency (b). 
(a)

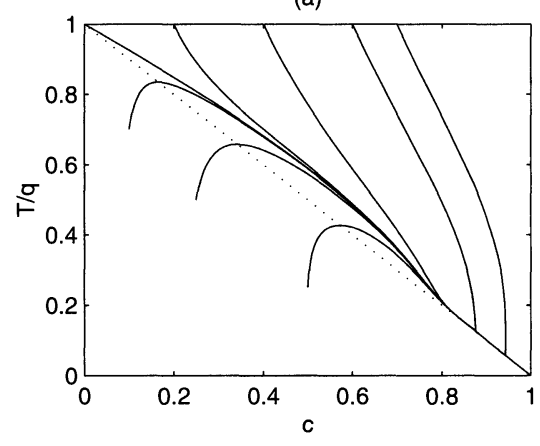

(c)

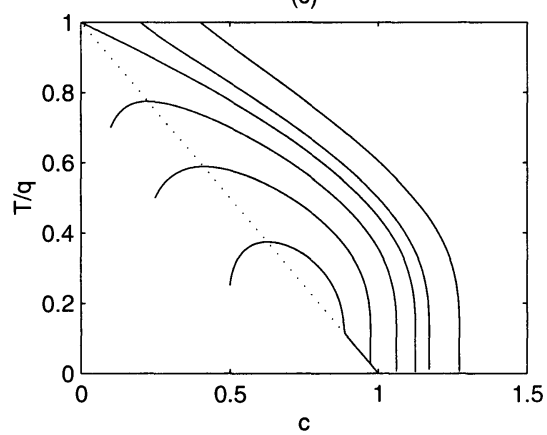

(b)

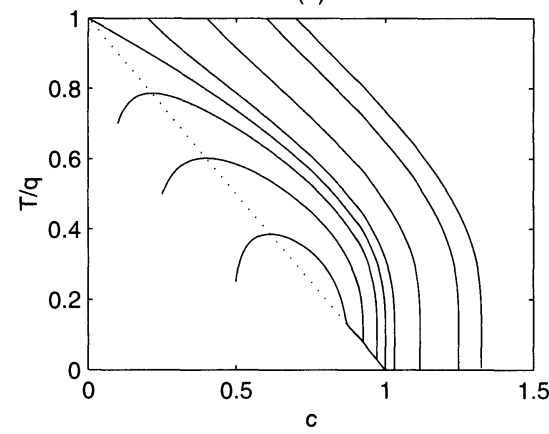

FIG. 2. Phase plane of (2.5) for $q=1$ and (a) $v=1>v^{*} \simeq .5707$, (b) $v=v^{*}$, and (c) $v=.5<v^{*}$.

This problem is the particular case for an infinite Lewis number of the problem treated numerically by Mikolaitis [12]. The exact relation

$$
T^{\prime}-v T=q v(c-1)
$$

accounts for conservation of the total enthalpy through the front and is readily obtained from (2.2)-(2.3). This relation, the second equality in (2.2), and a further elimination of the space variable allows us to reduce (2.2)-(2.3) to

$$
\begin{aligned}
& \frac{d T}{d c}=\frac{-q v^{2}[T+q(c-1)]}{c \exp (-1 / T)} \text { in } 0<c<1, \\
& T=q \quad \text { at } \quad c=0, \quad T=0 \quad \text { at } \quad c=1 .
\end{aligned}
$$

This problem is readily analyzed by phase plane methods as follows (see Figure 2 ). The relevant trajectories join the critical points $p_{1}:(c, T)=(0, q)$ and $p_{2}:(c, T)=(1,0)$. Point $p_{1}$ is a saddle with two incoming trajectories, namely, the $c=0$ axis and a trajectory with a negative slope of the form

$$
T=q-\frac{q^{2} v^{2} c}{q v^{2}+\exp (-1 / q)}+\cdots \quad \text { as } c \rightarrow 0 .
$$

In contrast, $p_{2}$ is a degenerate node, with infinitely many incoming trajectories which exhibit one of the following behaviors:

$$
T=q(1-c)+v^{-2} \exp \left[\frac{-1}{q(1-c)}\right]+\cdots
$$




$$
c=1-(q v)^{-2} \int_{0}^{T} u^{-1} \exp \left(\frac{-1}{u}\right) d u+\cdots
$$

as $c \rightarrow 1$. Note that the former behavior yields trajectories that are tangent to the straight line

$$
q c+T=q .
$$

Now, the problem (2.5)-(2.6) has a solution if and only if the nonvertical trajectory leaving the critical point $p_{1}$, hereafter called $\Gamma(v)$, passes through the critical point $p_{2}$. The following properties are readily proven (see Figure 2):

(a) $\Gamma(v)$ is above the straight line (2.10). This is so because $\Gamma(v)$ leaves $p_{1}$ along a direction with a higher slope than that of the straight line (see (2.7)) and cannot cross because the trajectories crossing this line exhibit zero slope.

(b) If, for some $v=v_{0}>0$, the trajectory $\Gamma\left(v_{0}\right)$ passes through $p_{2}$, then that trajectory also passes through $p_{2}$ for all $v>v_{0}$. This is so because the righthand sides of (2.5) and (2.7) strictly decrease as $v$ increases above the straight line (2.10). Thus $\Gamma(v)$ leaves $p_{1}$ between the straight line and $\Gamma\left(v_{0}\right)$ and can cross neither of them afterwards.

(c) For sufficiently large $v$, the trajectory $\Gamma(v)$ approaches the straight line (2.10) uniformly in $0<c<1$ and passes through $p_{2}$. That trajectory is given by

$$
T=q(1-c)+v^{-2} c \exp \left(\frac{-1}{q(1-c)}\right)+O\left(v^{-4}\right) .
$$

(d) For sufficiently small $v>0$, the trajectory $\Gamma(v)$ approaches the horizontal trajectory $T=q$ uniformly in $0<c<1$. Thus this trajectory does not pass through $p_{2}$.

(e) If $\Gamma\left(v_{1}\right)$ does not pass through $p_{2}$, then neither does $\Gamma(v)$ for $v_{1}<v<v_{1}+\varepsilon$ for some $\varepsilon>0$. This is a consequence of the continuous dependence on $v$ near $p_{2}$ of the trajectories that do not pass through $p_{2}$.

As a consequence of these properties, there is a critical value of the nondimensional burning rate, $v=v^{*}$, such that the problem (2.5)-(2.6) has a solution if and only if $v \geq v^{*}$; for $v \geq v^{*}$ the solution of (2.5)-(2.6) is unique. Note that, among the two possible asymptotic behaviors (2.8) and (2.9), the second one applies at the minimum burning rate $v^{*}$. And for each solution of $(2.5)-(2.6), T=T(c)$, there is an associated solution of $(2.2)-(2.3)$, whose concentration and temperature profiles are given by $c=c(\xi)$ and $T=T(c(\xi))$, where $c=c(\xi)$ is defined, up to translations, by

$$
\xi=q v \int_{c}^{0} c^{-1} \exp \left[\frac{1}{T(c)}\right] d c .
$$

Thus there are infinitely many plane fronts, one for each nondimensional burning rate in the range $v^{*} \leq v<\infty$.

If the nonlinearity in the right-hand side of (1.9) is slightly perturbed such that it vanishes if $0<T<\varepsilon$ for some $\varepsilon>0$, then, according to the phase plane arguments above, the resulting problem has a solution for one and only one value of $v, v_{\varepsilon}$; also $v_{\varepsilon} \rightarrow v^{*}$ as $\varepsilon \rightarrow 0$. Note that the situation is quite similar to that in adiabatic, premixed flames at unit $[13,14]$ and nonunit $[15,16,17]$ Lewis number; see also [18] for the nonadiabatic case. Therefore, only that plane front with the slowest burning 


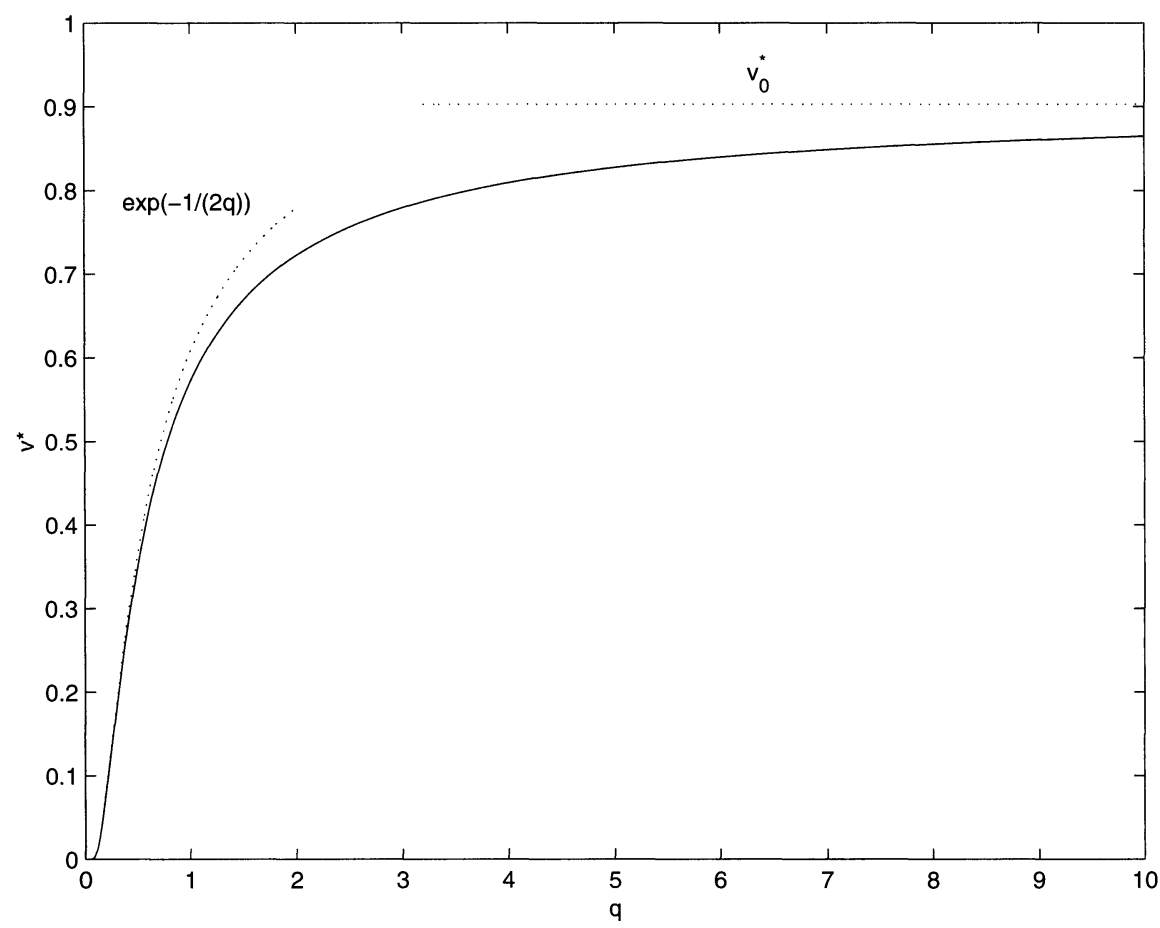

FIG. 3. The slowest burning rate of plane fronts in terms of the nondimensional heat of reaction; the asymptotic behaviors (2.12) and (2.13) are indicated with dotted lines.

rate $v=v^{*}$ survives under small perturbations of the problem, and thus only that solution will be considered below.

The burning rate $v^{*}$ is plotted vs. $q$ in Figure 3, and some representative temperature and reactant concentration profiles are given in Figure 4. Those curves are obtained by a standard shooting method from the cold boundary; the second equation in (2.2) and (2.4) are numerically integrated by a fourth order Runge-Kutta method with variable step, and the asymptotic behaviors (2.7) and (2.9) are used. The limits $q \rightarrow 0$ and $q \rightarrow \infty$ deserve some attention.

As $q \rightarrow 0$, the nondimensional burning rate is calculated by large-activationenergy asymptotics, as indicated in section 1 , to be

$$
v^{*}=\exp \left(\frac{-1}{2 q}\right)(1+O(q)) .
$$

As $q \rightarrow \infty$ we have

$$
v^{*}=v_{0}^{*}+O\left(\frac{1}{q}\right),
$$

where $v_{0}^{*}$ is uniquely given as the smallest value of $v^{*}$ such that the following problem has a solution:

$$
\begin{aligned}
& T^{\prime \prime}-v^{*} T^{\prime}=-\exp \left(\frac{-1}{T}\right) \quad \text { in }-\infty<\xi<\infty, \\
& T=0 \quad \text { at } \xi=-\infty, \quad\left|T-\frac{\xi}{v^{*}}\right|=\text { bounded } \quad \text { at } \xi=\infty .
\end{aligned}
$$



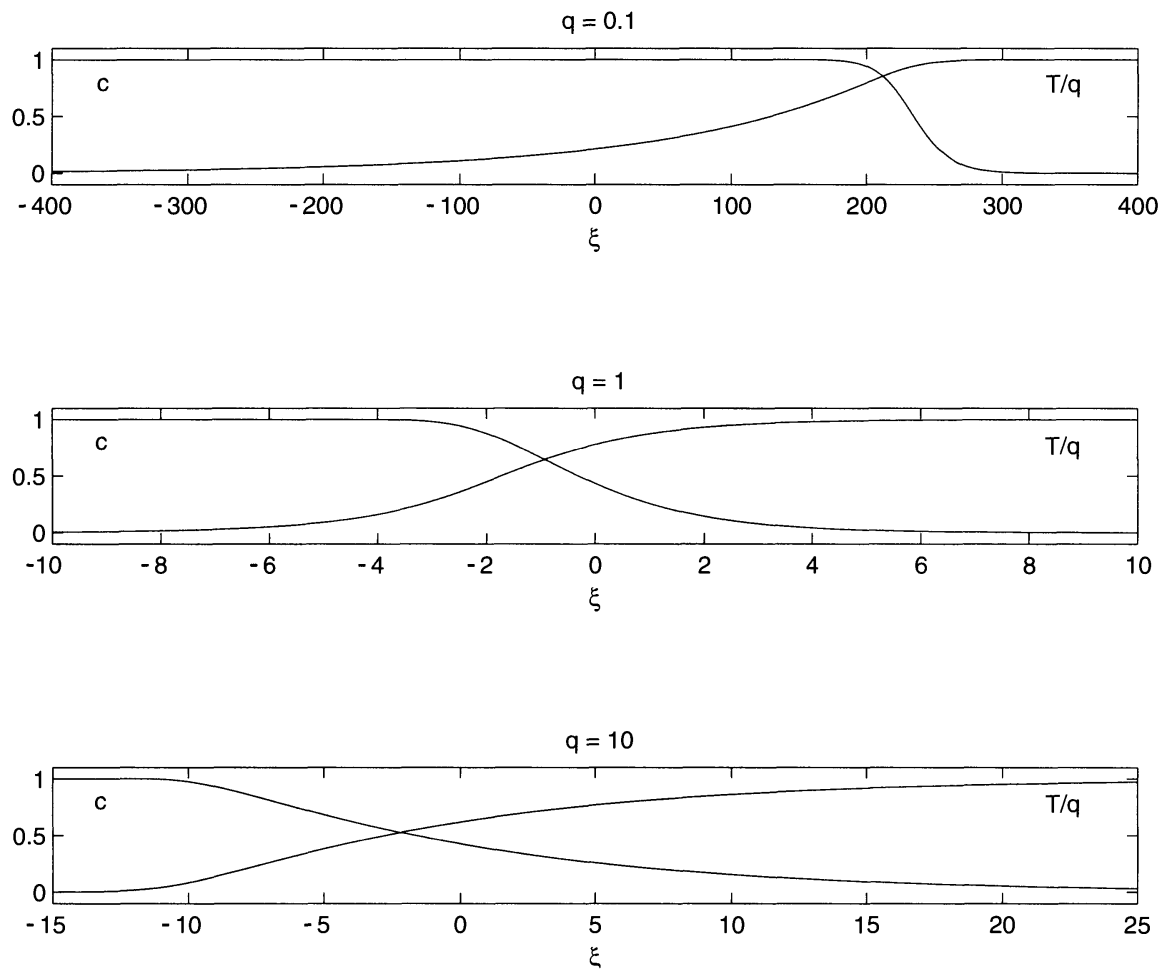

FIG. 4. Temperature and reactant concentration profiles for the plane fronts at the indicated values of the nondimensional reaction heat.

This problem applies in a first reaction zone where $|\xi| \sim 1$ and reactant consumption can be ignored. In addition there is a reaction tail, as $0<\xi \sim q$ where the reactant is consumed and the temperature increases to its final value $T=q . v_{0}^{*} \simeq 0.903$ is well defined, as obtained by phase plane arguments on the first order problem

$$
\begin{aligned}
& P \frac{d P}{d T}-v^{*} P=-\exp \left(\frac{-1}{T}\right) \quad \text { in } 0<T<\infty, \\
& P=0 \quad \text { at } T=0, \quad P=\frac{1}{v^{*}} \quad \text { at } T=\infty,
\end{aligned}
$$

to which (2.14)-(2.15) is reduced when defining $P=d T / d \xi$.

The asymptotic behaviors (2.12) and (2.13) are plotted with dotted lines in Figure 3. Note that the former is a fairly good approximation for not-so-small values of $\mathrm{q}$.

3. Linear stability of the plane front. We linearize (1.9)-(1.11) around a plane propagating front, $T=T(\xi), c=c(\xi)$, and consider only normal modes with a horizontal wavevector $\boldsymbol{k}$ (and wavenumber $k=|\boldsymbol{k}|$ ) of the form

$$
\hat{T}=\theta(\xi) \mathrm{e}^{\lambda t+\mathrm{i} \boldsymbol{k} \cdot \boldsymbol{y}}, \quad \hat{c}=\varphi(\xi) \mathrm{e}^{\lambda t+\mathrm{i} \boldsymbol{k} \cdot \boldsymbol{y}},
$$

with $\xi$ as defined in (1.14) and $\boldsymbol{y}=(y, z)$, and $\theta, \varphi$, and $\lambda$ as given by the linear eigenvalue problem 


$$
\begin{aligned}
& \theta^{\prime \prime}-v \theta^{\prime}+\frac{c \theta}{T^{2}} \exp \left(\frac{-1}{T}\right)+\varphi \exp \left(\frac{-1}{T}\right)=\left(\lambda+k^{2}\right) \theta \\
& -q v \varphi^{\prime}-\frac{c \theta}{T^{2}} \exp \left(\frac{-1}{T}\right)-\varphi \exp \left(\frac{-1}{T}\right)=q \lambda \varphi \\
& \theta \rightarrow 0, \varphi \rightarrow 0 \text { as } \xi \rightarrow \pm \infty .
\end{aligned}
$$

Here we consider only the point spectrum of (3.1)-(3.3). The continuous spectrum (under the reasonable assumption that $\theta$ and $\varphi$ are square integrable in $-\infty<\xi<\infty$ ) is readily seen using general results from [19] to exhibit negative real part; thus it does not lead to any instability. If (seeking for instability) we assume that the real part of $\lambda$ is nonnegative, $\Re \lambda \geq 0$, then the eigenfunctions decay exponentially as $\xi \rightarrow \pm \infty$, and both $\theta$ and $\varphi$ are in $L_{2}(\mathbb{R})$. Then, from (3.2)-(3.3) we obtain

$$
\varphi(\xi)=-(q v)^{-1} \exp \left[\frac{-\lambda \xi}{v}-\frac{h_{1}(\xi)}{(q v)}\right] \int_{-\infty}^{\xi} \exp \left[\frac{\lambda z}{v}+\frac{h_{1}(z)}{q v}\right] h_{2}(z) \theta(z) d z,
$$

where

$$
h_{1}(\xi)=\int_{-\infty}^{\xi} \exp \left[\frac{-1}{T(z)}\right] d z, \quad h_{2}(\xi)=\frac{c(\xi)}{T(\xi)^{2}} \exp \left[\frac{-1}{T(\xi)}\right] .
$$

Hölder's inequality yields

$$
|\varphi(\xi)| \leq(q v)^{-1} h_{3}(\xi)\|\theta\|_{L_{2}(\mathbb{R})},
$$

where

$$
h_{3}(\xi)=\left(\int_{-\infty}^{\xi} \exp \left[\frac{2 h_{1}(z)}{q v}\right] h_{2}(z)^{2} d z\right)^{1 / 2} \exp \left[\frac{-h_{1}(\xi)}{q v}\right] .
$$

On the other hand, multiplication of (3.1) by $\bar{\theta}$ (the complex conjugate of $\theta$ ) and integration on $-\infty<\xi<\infty$ yields

$$
\left(\lambda+k^{2}\right) \int_{-\infty}^{\infty}|\theta|^{2} d \xi=\int_{-\infty}^{\infty}\left[-\left|\theta^{\prime}\right|^{2}-v \bar{\theta} \theta^{\prime}+|\theta|^{2} h_{2}+\bar{\theta} \varphi \exp \left(\frac{-1}{T}\right)\right] d \xi
$$

where we have taken into account that $|\theta(\xi)|+\left|\theta^{\prime}(\xi)\right| \rightarrow 0$ exponentially as $\xi \rightarrow \pm \infty$. Since $\Re \int_{-\infty}^{\infty} \theta^{\prime} \bar{\theta} d \xi=\int_{-\infty}^{\infty}\left(\theta^{\prime} \bar{\theta}+\bar{\theta}^{\prime} \theta\right) d \xi / 2=0$, we need only to take the real part and the modulus in (3.5) and apply Hölder's inequality and (3.4) to obtain

$$
\Re \lambda+k^{2} \leq \delta_{1}+\frac{\sqrt{\delta_{2}}}{q v}, \quad\left|\lambda+k^{2}\right| \leq \frac{v}{4}+\delta_{1}+\frac{\sqrt{\delta_{2}}}{q v},
$$

where

$$
\delta_{1}=\max \left|h_{2}(\xi)\right| \text { for } \xi \in \mathbb{R}, \quad \delta_{2}=\int_{-\infty}^{\infty} \exp \left[\frac{-2}{T(\xi)}\right] h_{3}(\xi)^{2} d \xi
$$

Here we have taken into account that $\Re \lambda+k^{2} \geq 0$, that $\|\theta\|_{L_{2}(\mathbb{R})} \neq 0$, and the inequalities

$$
\left|v \int_{-\infty}^{\infty} \theta^{\prime} \bar{\theta} d \xi\right| \leq\left(\int_{-\infty}^{\infty}\left|\theta^{\prime}\right|^{2} d \xi \int_{-\infty}^{\infty} v|\theta|^{2} d \xi\right)^{1 / 2} \leq \int_{-\infty}^{\infty}\left|\theta^{\prime}\right|^{2} d \xi+\frac{v^{2}}{4} \int_{-\infty}^{\infty}|\theta|^{2} d \xi .
$$


Now, according to (3.6), both the real and the imaginary parts of $\lambda$ are bounded if the former is nonnegative. The first estimate in (3.6) provides an upper bound of the wavenumber at the instability threshold

$$
0 \leq k<k^{*}=\left(\delta_{1}+\frac{\sqrt{\delta_{2}}}{q v}\right)^{1 / 2} .
$$

In addition to giving bounds on the unstable eigenvalues, the estimates (3.6) show that the eigenvalues of (3.1)-(3.3) can come into the right-hand side of the complex plane only by crossing the imaginary axis. Note that this is trivially satisfied for finite Lewis number when the thermo-diffusive evolution equations are parabolic, but not here. The linear eigenvalue problem (3.1)-(3.3) is discretized by finite differences in the nonuniform mesh in which we calculated the steady state. We obtain the neutral instability curve (labelled "this paper") in Figure 1(a), whose points correspond to a pair of purely imaginary eigenvalues $\pm i \omega$; the eigenfrequency $\omega$ is also given in Figure 1(b). Note that the critical nondimensional heat release $q^{*} \simeq 0.1597$ is fairly small and not far from the threshold value in the strictly 1 -D case, $q_{0}^{*} \simeq 0.152$. As a consequence (see Figure 3), the associated burning rate is quite small, $v^{*} \simeq 0.0428$, which is in accordance with the asymptotic behavior (2.12). Also, the eigenfrequency at threshold $\omega^{*} \simeq 3.03 \cdot 10^{-3}$ and the associated wavenumber $k^{*} \simeq 2.0 \cdot 10^{-2}$ are quite small. This is due to the fact that $\left(q^{*}\right.$ is fairly small and) both $|\lambda|$ and $k^{2}$ depend exponentially on $-1 / q$ as $q \rightarrow 0$ (cf. (3.7)). Also note that the "exact" marginal instability curve, labelled this paper in Figure 1, significantly differs from that obtained by Buckmaster and Ludford using a delta-function model (labelled BL in Figure 1), albeit the comparison can be considered as qualitatively reasonable.

Let us now consider the large-activation-energy limit $q \rightarrow 0$ when there are exactly two eigenvalues with positive real part, which in fact are real and behave as

$$
\lambda_{1} q^{-1} \mathrm{e}^{1 / q} \rightarrow 2 \text { and } \lambda_{2} q^{2} \mathrm{e}^{1 / q} \rightarrow \Lambda_{0} \simeq 0.095 \text { as } q \rightarrow 0,
$$

where for simplicity we are considering only the restricted 1-D case. These two expressions are compared in Figure 5 with the exact unstable eigenvalues, numerically obtained as indicated above; in this figure we have plotted only real eigenvalues. In (3.7), $\lambda_{1}$ is obtained, after taking into account the rescaling (1.14), from the first equality of (1.16). Additionally, $\lambda_{2}$ is obtained from the reaction-zone equations

$$
\begin{aligned}
& \frac{\partial v}{\partial \sigma}=\frac{\partial^{2} v}{\partial \zeta^{2}}-w \mathrm{e}^{-v}, \quad \frac{\partial w}{\partial \sigma}=-\frac{\partial w}{\partial \zeta}-w \mathrm{e}^{-v} \quad \text { in }-\infty<\zeta<\infty \\
& v+\zeta=\text { bounded }, \quad w=1 \quad \text { as } \zeta \rightarrow-\infty, \quad v \rightarrow 0, w \rightarrow 0 \quad \text { as } \zeta \rightarrow \infty,
\end{aligned}
$$

in terms of the inner variables

$$
v=\frac{q-T}{q^{2}}, \quad \zeta=\frac{x \mathrm{e}^{1 /(2 q)}-t}{q}, \quad \text { and } \quad \sigma=t q^{-2} \mathrm{e}^{1 / q} .
$$

The boundary conditions (3.9) result from matching conditions with the outer solution, assumed stationary here. Equations (3.8)-(3.9) possess the steady state $w_{s}=$ $d v_{s} / d \zeta$, with $v_{s}=\ln [1+\exp (-\zeta)]$, which is unique up to translations. And we need only to linearize (3.8)-(3.9) around this steady state, seek modes in normal form, and solve numerically the resulting linear eigenvalue problem, whose growth rate is precisely the constant $\Lambda_{0}$ appearing in the second equation of (3.7). Note that $\lambda_{2}$ is associated with the dynamics in the reaction zone, as anticipated right after (1.16). 


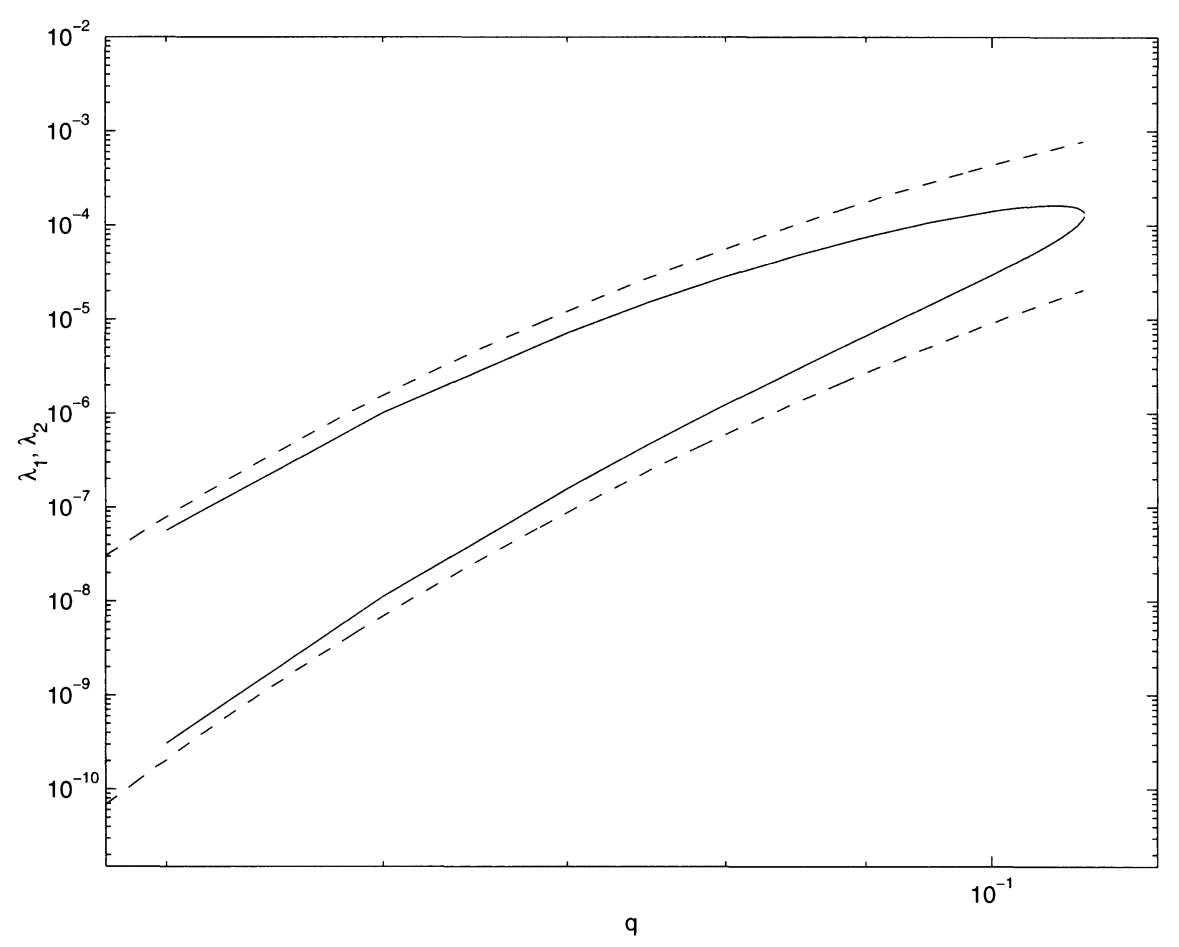

FIG. 5. Exact (-) and approximate (-- ), according to (3.7), unstable eigenvalues for small $q$.

4. Concluding remarks. We have considered a solid combustion model that applies in the limit of large heats of reaction. The problem depends only on one parameter, a conveniently nondimensionalized reaction heat $q$, which has been considered to be of order unity; the reaction is confined to a reaction sheet only in the limit $q \rightarrow 0$, which corresponds to a large Zel'dovich number. We have analyzed in section 2 the uniformly propagating plane fronts via phase plane arguments and shown that for each $q>0$ there is a critical value of the nondimensional burning rate $v^{*}$ such that for each $v>v^{*}$ there is exactly one (up to translations) front with a burning rate $v$, while for $v<v^{*}$ no front exists. Also, we have argued that only the slowest fronts (with a burning rate $v^{*}$ ) remain under perturbations of the reaction rate. The linear stability of the slowest front has been numerically analyzed in section 3. We have seen that this front is stable for $q>q^{*} \simeq 0.16$ and unstable otherwise, the instability being an oscillatory one, with a nonzero wavenumber. Thus, transversally propagating wavetrains (see, e.g., [20]) are to be expected near threshold.

Acknowledgment. We are indebted to Professor Amable Liñán for some useful discussions.

\section{REFERENCES}

[1] T. Boddington, A. Cottrell, And P.G. Laye, A numerical model of combustion in gasless pyrotechnic systems, Combustion and Flame, 76 (1989), pp. 63-69.

[2] P.A. Persson, R. Holmberg, And J. Lee, Rock Blasting and Explosives Engineering, CRC Press, Boca Raton, FL, 1994.

[3] F.A. Williams, Combustion Theory, Benjamin Cummings, San Francisco, CA, 1985. 
[4] A. BAYLiss AND B.J. MATKOWSKY, Fronts, relaxation oscillations and period doubling in solid fuel combustion, J. Comput. Phys., 71 (1987), pp. 147-168.

[5] B.J. MatKowsky AND G.I. Sivashinsky, Propagation of a pulsating reaction front in solid fuel combustion, SIAM J. Appl. Math., 35 (1978), pp. 465-478.

[6] N. Peters, On the stability of Liñan's premixed flame regime, Combustion and Flame, 33 (1978), pp. 315-318.

[7] D.S. STEWART AND J. BuckMASTER, The stability of Liñán's "premixed flame regime" revisited, SIAM J. Appl. Math., 46 (1986), pp. 582-587.

[8] D.S. STEWART, On the stability of the reaction zone of the plane deflagration, Combustion and Flame, 64 (1986), pp. 157-165.

[9] C. Alvarez Pereira AND J.M. VEgA, Global stability of a premixed reaction zone (timedependent Liñan's problem), SIAM J. Math. Anal., 21 (1990), pp. 884-904.

[10] J.D. Buckmaster ANd G.S.S. LudFord, Lectures on Mathematical Combustion, CBMS-NSF Regional Conf. Ser. in Appl. Math. 43, SIAM, Philadelphia, 1983.

[11] S.B. MARgOlis AND F.A. Williams, Diffusional/thermal instability of a solid propellant flame, SIAM J. Appl. Math., 49 (1989), pp. 1390-1420.

[12] D.W. Mikolaitis, Premixed flame structure for large activation energy and large heat release: A simple model, Combust. Sci. and Tech., 48 (1986), pp. 89-98.

[13] W.E. JOHNSON AND W. NACHBAR, Laminar flame theory and the steady linear burning of a monopropellant, Arch. Rational Mech. Anal., 12 (1963), pp. 58-92.

[14] W.E. Johnson, On a first-order boundary value problem from laminar flame theory, Arch. Rational Mech. Anal., 13 (1963), pp. 46-54.

[15] H. Berestycki, B. Nicolaenko, AND B. Scheurer, Traveling wave solutions to combustion models and their singular limits, SIAM J. Math. Anal., 16 (1985), pp. 1207-1242.

[16] M. MARION, Qualitative properties of a nonlinear system for laminar flames without ignition temperature, Nonlinear Anal., 11 (1985), pp. 1269-1292.

[17] M. MARION, Mathematical study of a model with no ignition temperature for laminar plane flames, in Reacting Flows: Combustion and Chemical Reactors, Part II, G.S.S. Ludford, ed., AMS, Providence, RI, 1986, pp. 239-252.

[18] V. Giovangigl, Nonadiabatic plane laminar flames and their singular limits, SIAM J. Math. Anal., 21 (1990), pp. 1305-1325.

[19] D. Henry, Geometric Theory of Semilinear Parabolic Equations, Springer-Verlag, New York, 1989.

[20] C. Alvarez-Pereira AND J.M. Vega, On the pulsating instability of two-dimensional flames, European J. Appl. Math., 3 (1992), pp. 55-73. 